samt zeigt die Studie, dass es immer wieder lohnend ist, sich mit statistischen Methoden die Wirksamkeit von Schmerzmedikamenten anzuschauen. So hat Paracetamol, das ja mal eines der beliebtesten Schmerzmedikamente überhaupt war, viel von sei- nem Ruf verloren, da es in vielen Studien und Metaanalysen bei klassischen Schmerzindikationen nicht besser als Placebo gewesen ist. Immerhin hat es noch einen Stellenwert etwa beim Kopfschmerz und wenn Kontraindikationen gegen andere der oben genannten Schmerzmedikamente vorliegen. Prof.Dr.Dr. Stefan Evers

Da Costa BR et al. Effectiveness of non-steroidal anti-inflammatory drugs for the treatment of pain in knee and hip osteoarthritis: a network meta-analysis. Lancet 2016; 387: 2093 - 105

\title{
Botulinumtoxin bei neuropathischem Schmerz ...
}

\section{Botulinumtoxin - BTX - erfreut sich inzwischen immer größerer Beliebtheit in der Schmerzmedizin. Eine Studie untersuchte die Wirkung bei neuropathi- schen Schmerzen - mit hoffnungsvollen Ergebnissen.}

D er Einsatz von BTX in einer Reihe von Indikationen ist inzwischen mit randomisierten kontrollierten Studien untersucht. In dieser placebokontrollierten Studie erhielten Patienten mit einer distal-symmetrischen schmerzhaften Polyneuropathie bis zu 300 E Ona-BTX A. Die Substanz wurde subkutan an FüBen und Unterschenkeln in zirka 30 Stellen injiziert. Die Injektionen erfolgten im Abstand von 12 über 24 Wochen. Insgesamt wurden in drei Zentren $152 \mathrm{~Pa}$ tienten eingeschlossen, von denen 66 in die primäre Analyse eingingen. Der primäre Endpunkt war die Reduktion der mittleren Schmerzintensität in einer Woche über den gesamten Behandlungs- zeitraum. Ona-BTX A führte dabei zu einer signifikant besseren Reduktion der neuropathischen Schmerzen als Placebo. Relevante Nebenwirkungen traten nicht auf. In der Analyse von Prädiktoren zeigte sich, dass eine Allodynie eine positive Wirkung vorhersagte.

\section{Kommentar}

Diese sehr hochrangig publizierte Studie ist ein weiterer Baustein zum Einsatz von BTX in der Schmerztherapie. Auf der einen Seite ist es folgerichtig, BTX auch beim neuropathischen Schmerz zu untersuchen, da es ja auch bei der Trigeminusneuralgie Hinweise auf eine Wirksamkeit gibt und wir aus anderen Studien wissen, dass die Wir- kung gegen Schmerzen sehr wahrscheinlich nichts mit der Wirkung an der motorischen Endplatte zu tun hat. Auf der anderen Seite muss man sich fragen, welchen Stellenwert BTX beim neuropathischen Schmerz haben wird, wenn die Ergebnisse dieser Studie repliziert werden können. In dieser Studie waren die Patienten relativ unselektiert eingeschlossen worden, auch die Ätiologie der Polyneuropathie spielte keine Rolle. Es muss also in zukünftigen Studien genauer darauf geachtet werden, welche Subgruppen mit neuropathischen Schmerzen eventuell von BTX profitieren. Ein Problem der Studie war auch, dass sehr viele Patienten nicht eingeschlossen werden konnten, es also viele Hinderungsgründe gab, BTX zu injizieren.

Prof. Dr. Dr. Stefan Evers

Attal N et al. Safety and efficacy of repeated injections of botulinum toxin $A$ in peripheral neuropathic pain (BOTNEP): a randomised, doubleblind, placebo-controlled trial. Lancet Neurol 2016; 15: 555-65

\section{... und beim chronischen Clusterkopfschmerz}

\author{
Die Injektion von Botulinumtoxin in das Ganglion sphenopalatinum könnte \\ eine Alternative zur Neurostimulation dieser Struktur werden.
}

$P$ atienten mit chronischem Clusterkopfschmerz sind oft schwer zu behandeln und zeigen sich nicht selten therapierefraktär. Bei ihnen sind in letzter Zeit neuromodulatorische Verfahren mit einigen Erfolgen ausprobiert worden. Insbesondere die Stimulation des Ganglion sphenopalatinum zeigte sich wirksam. Deswegen wurde jetzt beim chronischen Clusterkopfschmerz eine Injektion von BTX in diese Struktur ausprobiert. Dazu wurden in einer prospektiven, offenen und unkontrollierten Pilotstudie bei zehn Patienten mit therapierefraktärem Clusterkopfschmerz 25 oder 50 E Ona-BTX A in das Ganglion sphenopalatinum injiziert. Die Gabe erfolgte in Vollnarkose mittels eines neu entwickelten Injektionsgeräts.

Primärer Endpunkt war das Auftreten von Nebenwirkungen, was bei sieben der zehn Patienten der Fall war. Von den elf Ereignissen wurden vier als wahrscheinlich durch BTX ausgelöst gewertet (dreimal Akkomodationsstörung, einmal Schwäche der Kaumuskulatur), alle Ereignisse waren nach vier Wochen rückläufig. Nasenbluten kam ebenfalls vor. Die Wirksamkeit konnte nur bei sieben Patienten ermittelt wer- den. Die Attackenfrequenz pro Woche sank bei ihnen von $14 \pm 9$ auf $5 \pm 5$ ( $\mathrm{p}=$ 0,028). Die Attackendauer wurde durch die Injektion nicht beeinflusst.

\section{Kommentar}

Die BTX-Injektion in das Ganglion sphenopalatinum stellt eine interessante Möglichkeit dar, direkt in die Pathophysiologie des Clusterkopfschmerzes einzugreifen. Diese Studie zeigt erst einmal nur, dass bei den wenigen Patienten die Methode sicher und unproblematisch war. Die Wirksamkeit muss noch repliziert werden. Sollte dies gelingen, stellt dieses Verfahren eine Alternative zur Neurostimulation des Ganglion sphenopalatinum dar.

Prof. Dr.Dr. Stefan Evers

Bratbak DF et al. Pilot study of sphenopalatine injection of Ona-BTX A for the treatment of intractable chronic cluster headache. Cephalalgia 2016; 36: 503-9 